\title{
Schreibcoaching als Begleitinstrument im Promotionsprozess - Konzeptualisierung eines komplexen Interventionsformats
}

\author{
Vanessa Geuen $^{1}$ (D) - Ute Henning ${ }^{1}$ iD \\ Angenommen: 26. April 2021 / Online publiziert: 10. Juni 2021 \\ (c) Der/die Autor(en) 2021
}

\section{Zusammenfassung}

Was heißt und umfasst Schreibcoaching für Promovierende und welchen Beitrag kann es leisten, um den Promotionsprozess zielführend zu begleiten? Obwohl Schreibberatung und/oder Schreibcoaching für Promovierende an zahlreichen Universitäten im deutschsprachigen Raum seit Jahren praktiziert wird, liegt bislang kein umfassendes Konzept hierfür vor. In diesem Beitrag wird auf Basis einer Darstellung der wesentlichen Herausforderungen Promovierender beim Schreiben das Schreibcoaching als Begleitinstrument im Promotionsprozess theoretisch fundiert und konzeptionell reflektiert. Dazu werden verschiedene Beratungsansätze für Schreibberatung/-coaching vorgestellt, zueinander in Beziehung gesetzt und in Bezug auf das Schreibcoaching für die Zielgruppe der Promovierenden kritisch reflektiert. Die bislang weitgehend synonyme oder undifferenzierte Verwendung der Begriffe Schreibberatung und Schreibcoaching wird adressiert. Schreibcoaching wird als angemessenes Format der Begleitung Promovierender beim Schreiben vorgestellt.

Schlüsselwörter Schreibdidaktik · Dissertation · Promotion · Wissenschaftliches Schreiben · Schreibprozess · Schreibkompetenz

\section{Writing coaching as support for doctoral candidates - Conceptualization of a complex format}

\begin{abstract}
What does writing coaching for doctoral candidates mean, and how can it support the writing process of doctoral theses? Although many universities in German-speaking countries have been offering writing consultations and/or writing coaching to doctoral candidates for years, there is no comprehensive conceptualization of this format yet. Based on a discussion of some essential challenges that doctoral candidates face in their writing, this article proposes a theoretical and conceptual basis for writing coaching for doctoral candidates. To this end, we present, connect and critically discuss a number of approaches to writing consultation and coaching as far as they can serve the target group of doctoral candidates as coachees. We address the terms consultation and coaching, which as yet have mostly been used as synonyms or without proper differentiation, and we present our understanding of writing coaching as an adequate format for supporting doctoral candidates in their writing processes.
\end{abstract}

Keywords Teaching writing $\cdot$ Doctoral thesis $\cdot$ Graduate studies $\cdot$ Scientific writing $\cdot$ Writing process $\cdot$ Writing skills

Zweck/Ziel des Artikels Der Beitrag verortet Schreibcoaching für Promovierende anhand verschiedener Schreibberatungsansätze und stellt ihren Beitrag zur zielführenden Begleitung des Promotionsprozesses dar.

Hinweis zur ArticleCollection Interventionen im Schreibcoaching ID: AC_c114d12f0be7e54f7b03f3c07de9d366
Vanessa Geuen

vanessa.geuen@tu-darmstadt.de

Ute Henning

ute.henning@tu-darmstadt.de

1 SchreibCenter am Sprachenzentrum, TU Darmstadt, Hochschulstr. 1, 64289 Darmstadt, Deutschland 
Wetschanow und Fleischhacker (2020) konstatieren, dass der Begriff Schreibcoaching, ,nicht mehr aus der Praxis wegzudenken“ (ebd., S. 155) sei, sie machen aber auch auf die Unschärfen und unklaren Abgrenzungen des Formats Coaching allgemein und schließlich des Schreibcoachings aufmerksam, die sie auf unterschiedliche Gründe zurückführen (vgl. ebd., S. 156). In ihrem ,Versuch einer Begriffsdefinition" für Schreibcoaching nennen sie u.a. Klemm (2004), der den Unterschied zur Schreibberatung in der Intensität, Inhalts- bzw. Themenkomplexität und Dauer sehe (vgl. Wetschanow und Fleischhacker 2020, S. 157). In dieser Trias verorten auch wir uns im weitesten Sinne, wenn wir im folgenden Beitrag Schreibcoaching für Promovierende konzeptualisieren und - soweit möglich - theoretisch fundieren. Da wir kein allgemeines Konzept für Schreibcoaching vorlegen, sondern dieses für die Zielgruppe der Promovierenden definieren, beschreiben wir im ersten Kapitel die Herausforderungen und Schwierigkeiten beim Schreiben während der Promotion. Im zweiten Kapitel beleuchten wir unterschiedliche Perspektiven aus Schreibforschung und -didaktik und kombinieren diese mit Coaching- und Beratungstheorien. Schließlich bringen wir diese Aspekte zusammen und definieren das Spannungsfeld Schreibcoaching für Promovierende.

\section{Problemaufriss: Herausforderungen und Schwierigkeiten beim Schreiben während der Promotion}

Schreiben spielt für Promovierende eine zentrale Rolle, denn ihr Eintritt in die Wissenschaftsgemeinschaft erfolgt über Texte (vgl. Kruse 2007, S. 240; Reimers 2020; Par et al. 2009, S. 185-188): Sie erschreiben sich den Zugang in die Fachgemeinschaft mit der Dissertation (als Monografie oder über eine kumulative Promotion) und mit anderen Texten wie Zeitschriftenaufsätzen oder Konferenzbeiträgen. Auch darüber hinaus erfolgen wesentliche Teile ihrer Arbeit über schriftliche und mündliche Texte, für deren Erarbeitung das Schreiben wichtig ist, z. B. Vorträge, Korrespondenz, Drittmittelanträge usw. Immer mehr Promovierende schreiben ihre Dissertation und/oder andere Texte in der Wissenschaftssprache Englisch und somit in einer Fremdsprache (vgl. Brandt 2016, S. 1; Girgensohn und Liebetanz 2010, S. 181; Rabe 2016, S. 55f.; Reimers 2020, S. 10). Promovierende schreiben also viel und wollen möglichst effizient und mit erfolgreichem Ergebnis schreiben. Im Folgenden werden spezifische Herausforderungen, auf die sie beim Schreiben während der Promotion stoßen, und einige dadurch typischerweise entstehende Schwierigkeiten beschrieben. Neben der erwähnten Fachliteratur nutzen wir auch unsere Erfahrung aus dem Schreibcoaching und aus Schreibworkshops für Promovierende.
Man kann zwar davon ausgehen, dass Promovierende im Studium erfolgreich waren, dort Schreiberfahrungen gesammelt und erfolgreiche, d.h. gut benotete Leistungen erbracht haben (vgl. Girgensohn und Liebetanz 2010, S. 179). Aber daraus folgt nicht notwendigerweise, dass Promovierende souveräne Schreiber*innen sind oder während der Promotion mühelos schreiben werden. So bringen Promovierende in den MINT-Fächern (Mathematik, Informatik, Naturwissenschaft, Technik) vergleichsweise wenig Schreiberfahrung mit, da sie im Studium nur wenige zusammenhängende Texte schreiben mussten (vgl. Brandt 2016, S. 31; für den Maschinenbau Kuhn et al. 2016, S. 54 f.). Unabhängig von der Quantität der Schreiberfahrung aus dem Studium lässt sich diese jedoch ohnehin nicht ohne Weiteres auf das Schreiben einer Dissertation oder anderer wissenschaftlicher Texte während der Promotion übertragen, da hier die Anforderungen und die Schreibsituation anders sind (vgl. Girgensohn und Liebetanz 2010, S. 189). Besonders wenn Promovierende im Studium v.a. Klausuren geschrieben haben, ist offensichtlich, dass sie während der Promotionszeit ihre Schreibkompetenzen weiterentwickeln müssen (vgl. Dreyer 2006, S. $333 \mathrm{f}$.). Es kommt hinzu, dass gut benotete Texte (also Schreibprodukte) wie Studienabschlussarbeiten nicht auf eine in allen Facetten hohe Schreibkompetenz schließen lassen, da diese neben produktbezogenen (z.B. wissenschaftliche Ausdrucksfähigkeit) auch prozessbezogene Kompetenzen und Wissensbestände (z.B. Planungskompetenz, Selbstmotivation) beinhaltet (vgl. Kruse und Chitez 2014). Gerade in Bezug auf die Dissertation teilen unabhängig vom Fach die meisten unserer Coachees das Problem, dass die Arbeit an der Dissertation zwar immer wichtig, aber selten dringlich ist und neben den vielen anderen Aufgaben in der Lehre, der akademischen Selbstverwaltung usw. schnell in den Hintergrund tritt.

Zudem werden Promovierende mit veränderten Anforderungen an sich als Schreiber*innen konfrontiert, die herausfordernd wirken und immensen Druck auf sie ausüben können (vgl. Brandt 2016, S. 30-32; Fiedler und Hebecker 2006; Girgensohn und Liebetanz 2010, S. 180; Kaiser 2015). So unterscheidet sich eine Dissertation in zahlreichen Punkten von einer Master- oder einer anderen Studienabschlussarbeit. Zum einen sind Dissertationen schon aufgrund ihrer Länge anspruchsvolle Texte, was besonders für die Geistes- und Sozialwissenschaften gilt. Die Promovierenden arbeiten mehrere Jahre am großen Schreibprojekt Dissertation, was Durchhaltevermögen und die Fähigkeit zur Selbstmotivation sowie den langen und besonders komplexen Schreibprozess steuernde Kompetenzen erfordert. Gerade an mangelnder Selbststeuerung scheitert laut Fiedler und Hebecker (vgl. 2006, S. 242) so manche Promotion. Zum anderen müssen Dissertationen einen genuin neuen Beitrag zur Forschung enthalten - eine Anforderung, 
die so für studentische Arbeiten nicht gilt. Hinzu kommt, dass i.d. R. erwartet wird, zum Promotionsthema und darüber hinaus wissenschaftlich zu publizieren. Den wenigsten Promovierenden ist diese Aufgabe schon aus dem Studium bekannt und die Tatsache, dass man mit den eigenen Texten nun an die (Fach-)Öffentlichkeit treten muss, hat Auswirkungen auf den Schreibprozess.

Besonders während der Promotion besteht der Prozess des wissenschaftlichen Schreibens nicht bloß darin, den Verlauf einer Studie zu dokumentieren und Forschungsergebnisse festzuhalten. Vielmehr bedeutet Schreiben auch Erkenntnisproduktion, sodass der Schreibprozess eng mit dem Forschungsprozess verwoben ist (vgl. Fiedler und Hebecker 2006, S. 242). Dies ist vielen Promovierenden nicht bewusst. So sagen uns z.B. Promovierende aus der Chemie, dass sie erst im letzten halben Jahr ihrer Promotionszeit mit dem Schreiben ihrer Dissertationen anfangen. Dies führt wiederum dazu, dass sie das Schreiben einer größeren wissenschaftlichen Arbeit mit allen Herausforderungen in Bezug auf Kohärenz, Textstruktur, Adressatenorientierung usw. erst recht spät und wenig extensiv einüben. Während es in einigen MINT-Fächern nicht unüblich ist, erst im letzten Drittel der Promotionszeit oder noch später mit dem Schreiben an der Dissertation zu beginnen, führt ein später Schreibbeginn besonders in den Geisteswissenschaften zu enormem Zeitdruck und erschwert den Schreibprozess. So berichten auch Promovierende aus diesen Disziplinen häufig, dass sie in den ersten ein bis drei Jahren kaum zusammenhängende Textteile geschrieben, sondern vornehmlich gelesen und exzerpiert haben - dass auch diese Aktivitäten zum wissenschaftlichen Schreiben gehören und nutzbar gemacht werden können, reflektieren viele zunächst nicht. Des Weiteren können Schwierigkeiten im Forschungsprozess Auswirkungen auf den Schreibprozess haben, was umgekehrt ebenso gilt. Beispielsweise muss das Schreibprodukt Dissertation als linearer Text kohärent und klar strukturiert sein und den Forschungsprozess abbilden, während der Forschungsprozess selbst aber zirkulär abgelaufen sein mag (vgl. Dreyer 2006, S. 333).

Die genannten Herausforderungen führen nicht bei allen Promovierenden auch tatsächlich zu Schreibschwierigkeiten, denn Schreiben ist nicht nur generell ein äußerst individueller und von den unterschiedlich weit entwickelten Schreibkompetenzen geprägter Prozess, sondern die verschiedenen Fächer, Fachkulturen und Textsorten, in und an denen Promovierende schreiben, erhöhen diese Vielfalt weiter. In unseren Coachings kommen dementsprechend Schreibschwierigkeiten in ganz unterschiedlichen Aspekten des Schreibens zur Sprache. Die Promovierenden kommen etwa mit Unsicherheiten bei der Themenfindung und -eingrenzung, mit Fragen zum Umgang mit der Fülle an Literatur, die sie lesen wollen, oder mit Schwierigkeiten bei der effizienten Nutzung des Gelesenen für die eigenen
Texte. In diesem Zusammenhang sprechen Fiedler und Hebecker (2006, S. 238f.) auch von der Materialkrise: Nach dem von Enthusiasmus geprägten Beginn der Promotion setze bei vielen Promovierenden bei der Bearbeitung von bereits vorliegenden Theorien, Daten, Erkenntnissen usw. eine emotionale Krise ein, in der die Promovierenden sich von der Menge dieses Materials überfordert sehen. In diesem Stadium ist besonders wichtig, dass die Promovierenden ihr Promotionsthema, ihr Erkenntnisinteresse, ihre Forschungsfragen klar eingrenzen und formulieren. Nur dann können sie wieder zielorientiert am Text arbeiten, da Wichtiges von Unwichtigem unterschieden und Letzteres leichter aussortiert werden kann. Diesen Mut aufzubringen kann Promovierenden aller Fächer unserer Erfahrung nach schwerfallen.

Mit der Relevanzkrise bezeichnen Fiedler und Hebecker (2006, S. 239-241) eine umfassende Sinnkrise, bei der Promovierende alles in Frage stellen, nämlich die „Bedeutung der eigenen Forschungsergebnisse“ und den „Stellenwert im Vergleich zu anderen Arbeiten“ (ebd., S. 240) ebenso wie die Promotion an sich. Dies ist auch biografisch relevant, weil sich Promovierende grundsätzlich in ihrem Tun und Sein hinterfragen und weil sie im Hinblick auf ihre Zukunft nach der Promotion unsicher sind. Die Relevanzkrise kann auch damit in Verbindung gebracht werden, dass Promovierende in ihren Dissertationen und Publikationen schreiberische Expertise demonstrieren müssen, die sie in aller Regel nicht haben (können) (vgl. Casanave 2019): Die Dissertation wird zur Performance von Schreibkompetenz.

Die Ansprüche und Anforderungen, denen sich Promovierende ausgesetzt sehen und selbst aussetzen, sind häufig vage und sehr different. So stellen verschiedene Personen ganz verschiedene Ansprüche (z.B. Projektpartner*innen, Betreuer*innen, Kolleg*innen), die nicht immer transparent kommuniziert und erläutert werden und die einander widersprechen können. Die Promovierenden müssen zwischen all diesen ,Fremdansprüchen“ navigieren und Entscheidungen treffen, sie müssen permanent Prioritäten setzen. Auch innerhalb der wissenschaftlichen Fächer sind die Anforderungen unterschiedlich (vgl. z. B. Rabe 2016), sodass es kaum einen allgemeingültigen Kriterienkatalog geben kann, an dem sich Promovierende orientieren können. Neben diesen Anforderungen von außen kämpfen manche Promovierende zusätzlich mit kaum erreichbaren Ansprüchen an sich selbst, Perfektionismus kann sich auf alle Aktivitäten im Schreibprozess beziehen und darüber hinausgehen. Die Dissertation als akademisches Meisterstück wird häufig überfrachtet mit der Erwartung, in jeder Hinsicht das Bestmögliche aus sich herauszuholen und an die eigenen (z.B. intellektuellen) Grenzen zu gehen. Insgesamt kann das zur Abschlusskrise führen: Die Dissertation für fertig erklären zu müssen, sich mit dem eigenen Forschungsbeitrag $\mathrm{zu}$ exponieren und sich der Diskursgemeinschaft $\mathrm{zu}$ 
stellen, erfordert Mut und Selbstbewusstsein oder schlicht „ein entspanntes Verhältnis zum eigenen Produkt“ (Dreyer 2006, S. 334). Nicht zuletzt geht es in der Abschlusskrise auch um die Auseinandersetzung mit der Prüfungssituation, die das Einreichen der Dissertation bedeutet, und um die Unsicherheit der beruflichen Zukunft (vgl. Fiedler und Hebecker 2006, S. 242 f.).

Der Eintritt in die Fachgemeinschaft während der Promotion, bei dem das Schreiben und Publizieren wesentlich sind, kann als wissenschaftlicher Sozialisationsprozess verstanden werden, in dem die Doktormutter oder der Doktorvater (Betreuungsperson) eine zentrale Rolle spielt (vgl. Fiedler und Hebecker 2006, S. 249). Die Beziehung zur Betreuungsperson, die kaum formal geregelt ist, ist mitunter von Emotion und Projektion geprägt (vgl. Günther 2020, S. 103 f.), was die konstruktive Kommunikation und den sachlichen Umgang mit Konflikten erschwert. Darüber hinaus sind Betreuungspersonen in aller Regel nicht für Schreibschwierigkeiten sensibilisiert (vgl. Fiedler und Hebecker 2006, S. 249f.; Par et al. 2009, S. 183), sodass sie unserer Einschätzung nach eher eine Produktperspektive auf das Schreiben einnehmen. Für sie zählt das Ergebnis des Forschungs- und des Schreibprozesses, also der eingereichte und publizierte Text der Dissertation. Der Schreibprozess ist in den meisten Betreuungsbeziehungen kaum ein Thema. In unseren Schreibcoachings berichten Promovierende mitunter, dass sie sich auch gar nicht trauen, ihr Schreiben und mögliche Probleme dabei ihren Betreuungspersonen gegenüber zu erwähnen, weil sie glauben, dass das wissenschaftliche ,Schreiben-Können“ als selbstverständlich vorausgesetzt wird.

\section{Schreibberatungsansätze}

In diesem Kapitel beziehen wir uns auf wesentliche Grundannahmen und Prinzipien sowohl aus der Schreibforschung und Schreibdidaktik als auch aus Beratungs- und Coachingkonzepten. Ziel ist es, personenzentrierte, nondirektive Beratung den lösungsorientierten, systemischen Ansätzen gegenüberzustellen, die Anteile von Experten- und Prozessberatung zu benennen sowie Schreibberatung und Schreibcoaching vor diesen Hintergründen zu beschreiben.

\subsection{Personenzentrierte, nondirektive Schreibberatung}

Unser Verständnis von Schreibcoachings für Promovierende basiert einerseits auf dem personenzentrierten bzw. nondirektiven Ansatz nach Rogers (1972). Als einer der ersten fundierte der Schreibdidaktiker Gerd Bräuer das Format Schreibberatung sowohl auf schreibdidaktischer als auch auf gesprächspsychologischer Basis und richtete es an
Carl Rogers' humanistischem Menschenbild und damit an nondirektiven und personenzentrierten Beratungsansätzen aus. Mittlerweile hat sich diese Form der Schreibberatung im deutschsprachigen Hochschulraum weitgehend durchgesetzt. Andererseits legen wir systemische, d.h. lösungsorientierte Konzepte etwa nach Bamberger (2010) und Lange und Wiethoff (2014) zugrunde. Wir stellen zunächst Bräuers (2014) neun Grundprinzipien der Schreibberatung dar und setzen diese mit weiterer Grundlagenliteratur in Beziehung.

Auf Basis von Rogers' Annahme, dass Menschen ihre individuellen Lösungen in sich tragen und lediglich einer Begleitung beim Aufdecken dieser Lösungen bedürfen, ist das erste und wichtigste Prinzip von Schreibberatung bei Bräuer (vgl. 2014, S. 273) die Hilfe zur Selbsthilfe. Schreibberater*innen nehmen die Rolle von interessierten Zuhörenden und Lesenden ein, während die Verantwortung sowohl für das Beratungsgeschehen als auch für die Texte bei den Ratsuchenden bleibt. Als zweites Prinzip nennt Bräuer (vgl. 2014, S. 274f.) die Identifikation von Ursachen für Schreibschwierigkeiten, die er deswegen für zentral hält, weil Schreibende ihre Aufmerksamkeit häufig auf das Produkt, d.h. den Text und damit das Symptom, fokussierten. Letztlich müsse es aber darum gehen, sich den persönlichen Schreibprozess als komplexes und dynamisches System von zahlreichen Arbeitsschritten und Aktivitäten bewusst zu machen und in diesem Prozess an denjenigen Stellen anzusetzen, die Probleme verursachen. In der zugrundeliegenden prozessorientierten Schreibdidaktik wird der Schreibprozess entzerrt und in verschiedene Aktivitäten zergliedert, um Schreibende zu entlasten. Dazu gehören etwa Orientieren und Planen, Recherchieren, Material und Daten sammeln, Auswerten und Strukturieren, Schreiben (Rohfassungen und Textteile verfassen), Überarbeiten und Korrigieren (vgl. Kruse 2007, S. 110-115; Kruse und Ruhmann 2006). Diese Aktivitäten laufen im Schreibprozess nicht geordnet und nacheinander ab, denn Schreibende wechseln immer wieder zwischen den einzelnen Tätigkeiten, sodass jeder Schreibprozess individuell und rekursiv ist. Nach Dengscherz (2020) bewegen sich Schreibende von einer spezifischen Schreibsituation zur nächsten, bis der Text das von ihnen wahrgenommene Anforderungsniveau erreicht hat. Für Schreibberater*innen ist das Wissen um diese Komplexität und Dynamik von Schreibprozessen elementar, da die Schreibentwicklung ihrer Ratsuchenden, also „das Können und Wissen zur Textproduktion“ (Bräuer 2014, S. 258) Ausgangspunkt ihres aktuellen Schreibhandelns ist (vgl. auch Kellogg 2014, S. 130-136).

In einer Schreibberatung muss die Konzentration auf einen Beratungsschwerpunkt gelegt werden, so das dritte Prinzip nach Bräuer (vgl. 2014, S. 275). In einer Beratungssitzung können meist nicht alle Anliegen der Schreibenden oder alle im Schreibprozess entstandenen proble- 
matischen bzw. fehlenden Entscheidungen angemessen besprochen werden, sodass Berater*innen und Ratsuchende gemeinsam festlegen müssen, was sie zuerst bearbeiten. Diese Festlegung orientiert sich sinnvollerweise an dem Grundsatz HOC vor LOC (Higher Order Concerns vor Later Order Concerns), nach dem die zu bearbeitenden Textebenen hierarchisiert werden (vgl. Grieshammer et al. 2016, S. 103f., 110-112). Da die Ziele von Schreibberatung vorrangig ,,in einem besseren Text“ und in der ,gezielten Optimierung des [...] aktuellen [...] Schreibhandelns" bestehen (Bräuer 2014, S. 269), lautet Bräuers viertes Prinzip, eben jenes aktuelle Schreibhandeln zu priorisieren, um „den aktuell vorliegenden Schreibauftrag erfüllen zu helfen“ (ebd., S. 276). Erst in zweiter Linie oder eher indirekt werde damit auch die Schreibentwicklung gefördert. Prinzip fünf betrifft den Kompetenzbereich der Beratenden, der nicht verlassen werden dürfe, etwa wenn tieferliegende emotionale Probleme von Ratsuchenden zu Tage treten oder wenn fachliche Aspekte geklärt werden müssen, für die Betreuer*innen verantwortlich sind (vgl. ebd., S. 276). Prinzip sechs und sieben betreffen Aspekte der Vorbereitung und des sich Einlassens auf das Beratungsgespräch (vgl. ebd., S. 277 f.). Zentral hierbei ist das aktive Zuhören (u. a. mentale wie körperliche Zugewandtheit, Pacing, Spiegeln, Fragen, Empathie), aber auch das Bei-sich-Bleiben der Beratenden, also die Vermeidung von Projektionen eigener Erfahrungen auf die Ratsuchenden. Bräuer (ebd., S. 278) fokussiert außerdem drei relevante ,rhetorische Grundmuster", die allerdings in Interaktionsformen wie dem aktiven Zuhören und grundsätzlichen Gesprächsführungstechniken aufgehen: fragen statt feststellen, wahrnehmen statt interpretieren, antizipieren statt vorschreiben. Prinzip acht betrifft die Ausgewogenheit zwischen nondirektiven und direktiven Anteilen im Gespräch: Beratende sollten demnach ein Gespür dafür haben, wie viele Informationen und direktiven Input ein*e Ratsuchende*r benötigt und wo die/der Beratende sich zurückhalten sollte, ,um die ratsuchende Person wieder eigenverantwortlich handlungsfähig werden zu lassen“ (ebd., S. 279). Hiermit benennt Bräuer die Spannung zwischen Experten- und Prozessberatung, auf die wir in Kapitel 2.3 noch näher eingehen. Das neunte Prinzip verlangt die Einhaltung organisatorischer Regeln, von der Auftragsklärung über die gemeinsame Bestimmung des Beratungsschwerpunkts bis zur Nachbereitung des Gesprächs via Beratungsprotokoll etc. (vgl. ebd., S. 279f.).

Auch Grieshammer et al. (2016, S. 83-114) thematisieren in ihrem Grundlagenwerk Zukunftsmodell Schreibberatung sowohl Aufgaben als auch Grundsätze der Schreibberatung. Im Großen und Ganzen entsprechen diese Aufgaben und Grundsätze den neun Bräuer'schen Prinzipien. Besonders betonen die Autorinnen die personenzentrierte und die prozessorientierte Ausrichtung von Schreibberatung: Die Ratsuchenden stehen als autonome Personen und
Expert*innen für die jeweils passenden Lösungen im Mittelpunkt (vgl. Grieshammer et al. 2016, S. 98f.) und es gilt, ,sowohl den Schreibprozess im Ganzen zu sehen als auch die Schreibberatung als Prozess zu verstehen“ (ebd., S. 108).

\subsection{Lösungsorientierte, systemische Schreibberatung}

Die Schreibdidaktikerinnen Lange und Wiethoff (2014, S. 283) haben eine Einführung in die Systemische Schreibberatung vorgelegt, welche „als Alternative oder Ergänzung zur personenzentrierten (auch nicht-direktiven) Schreibberatung praktiziert wird" und als lösungsorientiert einzuordnen ist. Als systemisch bezeichnen sie ihre Konzeptualisierung zunächst, weil sie Schreibberatung im Kontext Wissenschaft verorten: „So findet sie in der Institution Universität statt, einem komplexen System mit speziellen Anforderungen und Konventionen; Schreiben ist als soziale Praxis immer auf Systeme (spezifische Diskursgemeinschaften) bezogen; Texte sind Teil solcher Gemeinschaften. Schreibende agieren in sozialen Systemen und können selbst als Systeme aufgefasst werden“ (Lange und Wiethoff 2014, S. 283 f.). Die lösungsorientierte Schreibberatung wird außerdem selbst als System verstanden, ,das aus verschiedenen Elementen besteht, die miteinander auf unterschiedliche Weise interagieren. Das System Schreiben ist ein Subsystem anderer Systeme, etwa des Kommunikationssystems oder des Systems Universität" (ebd., S. 285).

Schreibende werden in unterschiedlichen Systemen betrachtet, so etwa in ihrer Betreuungssituation, im Kontext der jeweiligen Erwartungen und Anforderungen, die an sie gestellt werden, oder es wird auch „,der zu schreibende Text im Diskurs der Forschungsmeinungen“ als System betrachtet, wobei man einzelne Aspekte nie sinnvoll isolieren könne, sondern immer den systemischen Kontext berücksichtigen müsse (vgl. ebd., S. 284f.). Das ist zwar richtig, aber auch die personenzentrierte Schreibberatung sieht dies so vor. So liegt neben dem anzufertigenden Produkt, dem Text, immer der Schreibprozess im Blick der Schreibberatenden. Nach dem Schreibprozessmodell von Hayes (2014) wird der Schreibprozess von äußeren Faktoren (Aufgabenumgebung) beeinflusst, die er in den sozialen Bereich (Adressat*innen, sog. Kollaborateur*innen) und den physischen Bereich (bisher geschriebene Texte bzw. Schreiberfahrungen, Schreibmedien) aufteilt (vgl. Hayes 2014, S. 62-64). Die inneren Einflüsse liegen beim Individuum und umfassen neben kognitiven Prozessen (Textinterpretation, Reflexion, Textproduktion) den Bereich der Motivation bzw. Emotion (Ziele, Einstellungen, Voraussetzungen) sowie das Langzeitgedächtnis (Handlungsmuster, thematisches, sprachliches, Wissen über Textsorten und Adressat*innen) (vgl. ebd., S. 64-83). Dieses System der 
unterschiedlichen Einflussfaktoren wird auch der personenzentrierten Schreibberatung zugrunde gelegt, nämlich wenn die bisherigen Schreiberfahrungen und die aktuelle Schreibsituation (z.B. Abgabedatum, Betreuungssituation) der Schreibenden oder Leser*innenlenkung und Erfüllung der geforderten Textsortenkonventionen thematisiert werden. Letztlich sind auch die oben beschriebenen Aktivitäten im Schreibprozess als System zu verstehen.

Schreiben ist also ein komplexer Prozess und damit ein dynamisches System. Und in der Schreibberatung geht es darum, diese Komplexität und Dynamik ,zu reduzieren, indem man [...] jeweils mit Ausschnitten aus dem Gesamtsystem bzw. mit der Konzentration auf einzelne Aspekte arbeitet. Dabei bleibt die Vielschichtigkeit des Schreibens und der Schreibenden erhalten, weil die jeweils ausgeblendeten Aspekte im Hintergrund präsent sind" (Lange und Wiethoff 2014, S. 285). Insofern fassen wir sowohl den personenzentrierten als auch den systemischen Ansatz als vornehmlich prozessorientierte Schreibberatung auf.

Weitere Gemeinsamkeiten und Ähnlichkeiten zwischen systemisch-lösungsorientierter und personenzentrierter Schreibberatung liegen in der möglichst hierarchiefreien, wertschätzenden und respektvollen Haltung der Beratenden gegenüber ihren Ratsuchenden (vgl. Lange und Wiethoff 2014, S. 284-287). Beide Konzepte basieren auf dem Prinzip der Hilfe zur Selbsthilfe, nach dem die Ratsuchenden selbst nachhaltige Lösungen für ihre aktuellen und künftigen Schreibprojekte finden. Die Autonomie der Ratsuchenden und damit deren Entscheidungsfreiheit und Verantwortung für ihre Texte und ihr Schreibhandeln macht die Schreibberatenden zu Begleiter*innen eines Prozesses, bei dem sie selbst keine eigenen Ziele verfolgen. Ratsuchende, ihre Schreibprozesse und Texte werden nicht beurteilt. Stattdessen ist die Aufdeckung und Nutzbarmachung ihrer Ressourcen und Kompetenzen und damit die Vergrößerung ihrer Handlungsoptionen übergeordnetes Ziel von Schreibberatung.

Ein Unterschied zwischen personenzentrierter und systemisch-lösungsorientierter Schreibberatung besteht im Stellenwert der Ursachen für Schreibprobleme. So hält Bräuer (2014, S. 274) das ,Aufdecken der eigentlichen Ursache für wahrgenommene Reibungsverluste in der Textproduktion" für eine zentrale Aufgabe der Schreibberatung. Es sei „äußerst wichtig, herauszufinden“, ob die Ursachen für Schreibschwierigkeiten, die Schreibende benennen und selbst identifiziert haben, ,vielleicht in einer früheren Phase der Textproduktion“ liegen und somit den weiteren Verlauf des Schreibprozesses hemmen bzw. negativ beeinflussen. Auch Grieshammer et al. (2016, S. 92) betonen diesen Aspekt, da Schreibende ihre Schwierigkeiten zwar meist registrierten, häufig aber nicht verstünden, worin sie genau bestehen und wo ihre Ursachen liegen. Beim systemischen Ansatz dagegen kommt es laut Bamberger (2010,
S. 41) es nicht auf die Kenntnis der Problemursachen an. In der systemischen Schreibberatung sei es nicht die Aufgabe der Beratenden, „Ursachen von Schreibproblemen [zu] verstehen oder [zu] ergründen" (Lange und Wiethoff 2014, S. 286). Was auf den ersten Blick als entgegengesetzte Ansätze für Schreibberatung erscheint, zeigt sich bei der genaueren Analyse aber doch als nahe beieinander liegend: Wenn Bräuer (2014, S. 274f.), Grieshammer et al. (2016, S. 92 f.) und andere Vertreter*innen der personenzentrierten Schreibberatung die Relevanz von Problemursachen betonen, geht es dabei doch immer darum, diese als Ausgangspunkt für Lösungsstrategien zu betrachten. Auch Bamberger (2010, S. 41f.) als einer der bekanntesten Vertreter der systemischen Beratung betrachtet „Probleme als Impulsgeber“, ,als etwas völlig Normales“, als Möglichkeit, die „den Menschen [überhaupt erst] veränderungsfähig“ mache, und als Ausgangspunkt ,für Entwicklung und persönliches Wachstum“. Lösungsorientierung meint in diesem Zusammenhang Zukunftsorientierung, Horizonterweiterung und Perspektivwechsel und zielt auf die Vergrößerung von Handlungs- und Gestaltungsspielräumen durch das Aufdecken von Ressourcen ab (vgl. ebd., S. 43-45). Dabei werden die Ursachen für etwaige Probleme allerdings als irrelevant eingestuft.

\subsection{Experten- vs. Prozessberatung}

Es herrscht Einigkeit darüber, dass weder die personenzentrierte noch die lösungsorientierte Beratung reine Prozessberatung sein kann. Schreyögg (2010, S. 130) legt überzeugend dar, dass in personenzentrierten Beratungs- bzw. Coaching-Settings ,die Prozessberatung [...] durch Sequenzen von Expertenberatung [...] angereichert werden“" muss (vgl. auch König und Volmer 2019, S. 16). Und Bamberger (2010, S. 48) beschreibt die Beziehung zwischen Berater*in und Klient*in als ,kollaboratives Expertensystem“, in dem „das Expertenwissen von Berater und Klient wirksam ist“.

Im Kontext von Schreibberatung kommen wir hier auf das achte Prinzip von Bräuer (2014) zurück, das ebenfalls die Mischung aus Experten- und Prozessberatung betrifft. Bräuer beschreibt das koproduktive Wesen von Schreibberatung, die ,in einem dynamischen Spannungsgefüge von nicht-direktiver und direktiver Beratungsmethode“" stattfinde (Bräuer 2014, S. 269). Zwar muss die Verantwortung sowohl für die Texte als auch das Schreibhandeln immer bei den Ratsuchenden bleiben (vgl. Girgensohn und Liebe$\operatorname{tanz} 2010$, S. 186). Doch wird die Expertise von Schreibberatenden bezüglich schreibdidaktischer Inhalte fruchtbar gemacht, um ,das bei der ratsuchenden Person vorhandene Potenzial zur [...] Selbststeuerung auszuloten und $\mathrm{zu}$ aktivieren“ und einen ,gemeinsam definierte[n] Aktionsraum“ zu gestalten (Bräuer 2014, S. 273). Das fachsensible Schreibberatungsmodell von Keding und Scharlau (2016), 
mit dem sie das Spannungsfeld zwischen fächerübergreifender und fachspezifischer Schreibberatung beleuchten, nutzt eine ähnliche Mischform aus Experten- und Prozessberatung. Zentral ist auch hier, dass es in der Schreibberatung „nicht um ein Entweder-Oder“ zwischen Experten- und Prozessberatung gehe, sondern dass die Beratenden ,sozusagen in Personalunion beide Beratungsformen anwenden“ müssten (ebd., S. 120).

\subsection{Schreibberatung und Schreibcoaching}

Es zeigen sich zahlreiche Gemeinsamkeiten zwischen Schreibberatung und Schreibcoaching: Beide Konzepte basieren auf demselben Interaktionsstil, der u.a. die Mischung zwischen Experten- und Prozessberatungsanteilen, Personenzentrierung und Lösungsorientierung beinhaltet (vgl. Bräuer 2014; Lange und Wiethoff 2014; Keding und Scharlau 2016; Nußbeck 2010, S. 18-21; Rogers 1972; Schreyögg 2012, S. 209f.). Grundsätzlich müssen Schreibberatung und Schreibcoaching insofern Prozessberatungen sein, weil sie erstens den Schreibprozess fokussieren. Zweitens sind sie personenzentriert und lösungsorientiert ausgerichtet, da die Schreibenden im Mittelpunkt stehen und ihre individuellen Lösungen selbst finden sollen. Das Prinzip Hilfe zur Selbsthilfe wird in allen schreibdidaktischen Publikationen und von entsprechend ausgerichteten Schreibzentren zum obersten Grundsatz erhoben. Unseres Erachtens gilt jedoch auch in diesem Kontext, dass die reine nondirektive Prozessberatung dort ihre Grenzen hat, wo Schreibberater*innen und Schreibcoaches als Expert*innen für schreibdidaktische Erkenntnisse und Prozesse, für Wissenschaftssysteme und die Unterschiede zwischen den Disziplinen sowie für die besonderen Bedingungen des Promovierens auftreten müssen.

Gerd Bräuer ist innerhalb der Schreibforschung und Schreibdidaktik bisher einer der Wenigen, die explizit Schreibberatung von Schreibcoaching abgrenzen. Er bezeichnet Schreibberatung als, individuelle Begleitung von Schreibenden in allen Phasen der Textproduktion durch ausgebildete Schreibberater*innen auf der Grundlage des Prinzips ,Hilfe zur Selbsthilfe““ (Bräuer 2014, S. 269). Das Ziel von Schreibberatung sieht er v.a. ,in einem besseren Text"“, in der ,gezielten Optimierung des [...] aktuellen [...] Schreibhandelns“ und damit in einem konkreten „Beitrag zur Schreibentwicklung“ (ebd., S. 269). Schreibcoaching dagegen bezeichne ,das Umsetzen eines Trainingsprogramms zur gezielten Veränderung des aktuellen Schreibhandelns [...] unter längerfristiger Anleitung durch eine mit dem Trainingsprogramm vertraute Fachperson" (ebd., S. 270). Die Beratung ist hier also eher als punktuelle Unterstützung für konkrete und aktuelle Schreibprojekte zu verstehen, gleichwohl aus ihr nachhaltige Veränderungen und Verbesserungen des individuellen Schreibhandelns erwachsen können und sollen. Das Coaching umfasst von vornherein mehrere Beratungssitzungen, die mehr oder weniger eng ineinandergreifen und aufeinander aufbauen. Somit können umfassendere Themen, Anliegen, Fragen und Probleme bearbeitet werden und die Arbeit an einem umfangreicheren Schreibprojekt kann leichter prozessorientiert und die Schreibentwicklung unterstützend begleitet werden. Einen weiteren Vorschlag zur Unterscheidung von Schreibberatung und -coaching macht Fröchling (2018), die der Schreibberatung abspricht, einen Prozess zu begleiten (vgl. ebd., S. 19). Sie sieht in der Schreibberatung - in Abgrenzung zum Schreibcoaching - „Fragen zur Textproduktion [in Form von] Rat, Information und Tipps“ im Fokus und unterscheidet die Schreibberater*in als „Ratgeber*in“ vom Schreibcoach als „Prozessbegleiter*in“ (Fröchling et al. 2020, S. 164). Und in Kuntschners (2020) Verständnis ,ist Schreibberatung eine bestimmte Form der Fach-Beratung und fokussiert auf die Fachinhalte und auf den Schreibprozess. Schreibcoaching hingegen fügt dem noch den Aspekt der Prozessberatung hinzu, die auf Rollenreflexion [der Coachees] abzielt"،. Diese beiden Differenzierungsversuche sehen wir kritisch, wie aus unseren obigen Ausführungen ersichtlich geworden sein dürfte.

\section{Unser Konzept von Schreibcoaching für Promovierende}

Im Folgenden stellen wir auf Basis der bisherigen Ausführungen unser Konzept von Schreibcoaching für Promovierende vor. Dabei ist zu beachten, dass dieses Konzept nicht als allgemeine Definition von Schreibcoaching zu verstehen ist, sondern sich wesentlich durch die Zielgruppe der Promovierenden definiert. Den Begriff Schreibcoaching verwenden wir im Bewusstsein, dass sich unser Konzept in vielerlei Hinsicht mit oben diskutierten Auffassungen von Schreibberatung deckt, an manchen Punkten aber auch Unterschiede $\mathrm{zu}$ erkennen sind.

Wie erwähnt ist die Promotion von einem mit dem Schreiben verwobenen Forschungsprozess geprägt, der weit über das Anfertigen der Dissertation hinausgeht. Das Schreiben während der Promotion ist soziales Handeln (vgl. Keding und Scharlau 2016, S. 123) in dem speziellen System der Wissenschaft.

Das bedeutet zunächst einmal, Schreibcoaching für Promovierende als kooperative Beziehung zwischen Schreibcoach und Coachee zu verstehen, in der es um eine gemeinsame Reflexion über das Schreiben im weitesten Sinne geht (vgl. hierzu den Ausdruck „Arbeitsbündnis“ von Kuntschner 2020). Schreibcoaching für Promovierende ist personenzentriert, lösungs- und ressourcenorientiert, d.h. die Promovierenden und ihre Verortung in Schreibprozessen und Disziplinen mit den jeweils herrschenden Stan- 
dards, Konventionen und Textsorten stehen im Mittelpunkt und sie entscheiden mit, wie stark sie ihre Textprodukte ins Coaching einbringen. Ihre Schreibkompetenzen und individuellen Schreibstrategien (s. hierzu Kap. 1) werden nutzbar gemacht, um einerseits Schreibschwierigkeiten zu bewältigen und andererseits die Handlungsfelder und Gestaltungsspielräume zu vergrößern.

Schreibcoaches sind die Expert*innen für schreibdidaktische Hintergründe und Interventionen (wie etwa Schreibtechniken, -übungen, -methoden), für beraterisches Handeln und Gesprächsführungstechniken und für das System Wissenschaft, z.B. dessen Kommunikationsgepflogenheiten, dessen zumindest im deutschsprachigen Raum hierarchische Sozialstrukturen, dessen Regularien wie Promotionsordnungen. Da das Schreibcoaching für Promovierende an unserer Universität ein fächerübergreifendes Angebot ist, sind Schreibcoaches darüber hinaus Expert*innen für fachsensibles Agieren, d.h. sie wissen um die Diversität der Disziplinen und ihrer Anforderungen, Konventionen, Regeln. Die Promovierenden wiederum sind die Expert*innen für ihre individuellen Schreibprozesse und Schreibstrategien, ihre Disziplinen und ihre Inhalte, sodass auch von ihrer Seite fachliches und systemisches Expert*innenwissen in die Sitzungen einfließt. Damit spielt sich Schreibcoaching für Promovierende zwischen Experten- und Prozessberatung ab.

Schreibcoaching für Promovierende erfordert eine fundierte schreibdidaktische Ausbildung der Schreibcoaches, die Wissen über Schreibprozesse, Schreibstrategien, Schreibtypen, Schreibkompetenzen, Textsorten, Wissenschaftskulturen ebenso beinhaltet wie die praktische Nutzung von Gesprächsführungstechniken, Interaktionsstilen usw. (vgl. u. a. Bräuer 2014; Girgensohn und Sennewald 2012; Girgensohn und Liebetanz 2010; Grieshammer et al. 2016). Zusätzlich sollten die Schreibcoaches über Kenntnisse von Beratungs- bzw. Coachinggrundlagen verfügen. Auch sollten sie u.E. selbst promovieren oder promoviert haben, weil sie nur so die speziellen Rahmenbedingungen und veränderten Arbeits- und Schreibprozesse der Promovierenden wirklich nachvollziehen und sich darauf einstellen können. Nicht zuletzt sollten sie breites Wissen über die Institution, d.h. das System Universität haben, in dem so spezifische und komplexe, von außen kaum durchschaubare Strukturen etabliert sind. In manchem Coachingratgeber heißt es zwar, die beste Ausgangssituation für Coaches sei, über möglichst wenig Kenntnisse der Arbeits- und Lebensbedingungen ihrer Coachees zu verfügen. Doch sehen wir diese Behauptung insbesondere bei Promovierenden kritisch, da die Wissenschaftslandschaft so eigenen geschriebenen wie ungeschriebenen Gesetzen unterliegt.

Da wir oben Schreibcoaching für Promovierende als kooperative Beziehung definiert haben, ergeben sich da- raus automatisch zwei weitere Unterscheidungsmerkmale zu Schreibberatung: Erstens wird ein Schreibcoaching von vornherein auf mehrere Sitzungen angelegt, es kann - im Gegensatz zu Schreibberatung - nicht nur eine Sitzung umfassen, in der kaum eine Beziehung entstehen kann. Promovierende werden im Schreibcoaching während eines Arbeitsprozesses begleitet, der sich über mehrere Jahre erstreckt und bei dem entsprechend komplexe Schreibprozesse so entzerrt werden können, dass auch ihre besondere Dynamik durch regelmäßige und den jeweiligen Bedarfen angepasste Coachings strukturiert werden kann. Zweitens finden Schreibberatungen oft nach dem Drop-in-Prinzip ohne Anmeldung und bei wechselnden Schreibberater*innen statt. Für Schreibcoachings sind beide Konzepte eher ungeeignet (vgl. hierzu auch Girgensohn und Liebetanz 2010, S. 188). Nur verbindliche Terminvereinbarungen gewährleisten einen kontinuierlichen Coachingprozess. Dafür ist auch notwendig, dass dieselben Schreibcoaches sich gemeinsam mit den Promovierenden auf den Auftrag bzw. die Inhalte und den Zeitraum des Coachings einigen. Würden die Schreibcoaches wechseln, wäre der Aufbau einer vertrauensvollen Beziehung nicht möglich.

Die Promotionszeit ist wie in Kap. 1 erläutert vom Schreiben verschiedenster Texte mit unterschiedlichen Zielen, Adressat*innen und Inhalten geprägt. Die z.B. daraus resultierende Auseinandersetzung mit unterschiedlichen Textsorten, die jeweils angewendeten Schreibstrategien, zu knappe Zeitplanung bei einem Schreibprojekt und andere Aspekte des Schreibprozesses können im Schreibcoaching reflektiert und lösungs- und ressourcenorientiert nutzbar gemacht werden für das Großprojekt Dissertation.

\section{Resümee}

Um unsere Konzeptualisierung von Schreibcoachings Promovierender und die in den vorigen Abschnitten beschriebenen Aspekte zusammenzufassen, nutzen wir die folgende Definition von (systemischem) Coaching von Webers (2020, S. 3), die wir teilweise wörtlich übernehmen und für das Schreibcoaching für Promovierende anpassen: Schreibcoaching fördert die Fähigkeit Promovierender zur Selbstorganisation auf Ebene des professionellen, d.h. wissenschaftlichen Schreibens. Schreibcoaching unterstützt Promovierende bei der Gestaltung ihrer persönlichen Schreibentwicklung, ihrer sozialen Rollen beim Schreiben bzw. in ihren Texten und ihrer Kooperationsbeziehungen innerhalb ihrer wissenschaftlichen Diskursgemeinschaften sowie bei der Bewältigung ihrer Entscheidungs- und Handlungsanforderungen während der Promotion. In der Zusammenarbeit zwischen Schreibcoach und Promovierende*r werden Reflexionsräume eröffnet und Klärungsprozesse initiiert (etwa im Hinblick auf Schreibprozesse, individuelle 
Schreibstrategien und Schreibschwierigkeiten). Durch die Erschließung neuer Perspektiven werden Entwicklungspotenziale und Handlungsspielräume für aktuelles und künftiges Schreibhandeln erschlossen und Lern- und Veränderungsprozesse angeregt.

Open Access Dieser Artikel wird unter der Creative Commons Namensnennung 4.0 International Lizenz veröffentlicht, welche die Nutzung, Vervielfältigung, Bearbeitung, Verbreitung und Wiedergabe in jeglichem Medium und Format erlaubt, sofern Sie den/die ursprünglichen Autor(en) und die Quelle ordnungsgemäß nennen, einen Link zur Creative Commons Lizenz beifügen und angeben, ob Änderungen vorgenommen wurden.

Die in diesem Artikel enthaltenen Bilder und sonstiges Drittmaterial unterliegen ebenfalls der genannten Creative Commons Lizenz, sofern sich aus der Abbildungslegende nichts anderes ergibt. Sofern das betreffende Material nicht unter der genannten Creative Commons Lizenz steht und die betreffende Handlung nicht nach gesetzlichen Vorschriften erlaubt ist, ist für die oben aufgeführten Weiterverwendungen des Materials die Einwilligung des jeweiligen Rechteinhabers einzuholen.

Weitere Details zur Lizenz entnehmen Sie bitte der Lizenzinformation auf http://creativecommons.org/licenses/by/4.0/deed.de.

\section{Literatur}

Bamberger, G. (2010). Lösungsorientierte Beratung: Praxishandbuch. 4., vollständig überarbeitete Aufl. Weinheim: Beltz.

Brandt, A. (2016). Help me write it right: Eine empirische Analyse individueller Schreibcoachings für Promovierende. Bochum: AKS.

Bräuer, G. (2014). Grundprinzipien der Schreibberatung: Eine pragmatische Sicht auf die Schreibprozesstheorie. In S. Dreyfürst \& N. Sennewald (Hrsg.), Schreiben: Grundlagentexte zur Theorie, Didaktik und Beratung (S. 257-282). Opladen: Barbara Budrich.

Casanave, C.P. (2019). Performing expertise in doctoral dissertations: Thoughts on a fundamental dilemma facing doctoral students and their supervisors. Journal of Second Language Writing, 43, 57-62. https://doi.org/10.1016/j.jslw.2018.02.005.

Dengscherz, S. (2020). Professionelles Schreiben in mehreren Sprachen - das PROSIMS-Schreibprozessmodell. Zeitschrift für Interkulturellen Fremdsprachenunterricht, 25(1), 397-422. https:// tujournals.ulb.tu-darmstadt.de/index.php/zif/article/view/1023. Gesehen 20.03.2021.

Dreyer, E. (2006). Schreibprobleme? Eine exemplarische Übersicht, wie Promovierende diese überwinden können. In C. Koepernik, J. Moes \& S. Tiefel (Hrsg.), GEW-Handbuch Promovieren mit Perspektive: Ein Ratgeber von und für DoktorandInnen (S. 332-338). Bielefeld: Bertelsmann.

Fiedler, W., \& Hebecker, E. (2006). Promotionskrisen und ihre Bewältigung. In C. Koepernik, J. Moes \& S. Tiefel (Hrsg.), GEWHandbuch Promovieren mit Perspektive: Ein Ratgeber von und für DoktorandInnen (S. 236-251). Bielefeld: Bertelsmann.

Fröchling, A. (2018). Professionelles Schreibcoaching: Konzept, Methoden, Praxis. Hamburg: Tredition.

Fröchling, A., Fleischhacker, M., Wetschanow, K. \& Mertlitsch, C. (2020). Schreibcoachs arbeiten ,ganzheitlich, mit Methoden aus der Psychologie und dem Führungskräftecoaching“ - über Entwicklungen, Einflussfaktoren und Qualitätskriterien im Schreibcoaching: Ein Praxisdialog mit Anke Fröchling. Coaching | Theorie \& Praxis 6(1): 163-166.

Girgensohn, K., \& Liebetanz, F. (2010). Schreibberatung für inund ausländische Promovierende als institutionelles Angebot. In K. Girgensohn (Hrsg.), Kompetent zum Doktortitel: Konzepte zur Förderung Promovierender (S. 179-197). Wiesbaden: VS.
Girgensohn, K., \& Sennewald, N. (2012). Schreiben lehren, Schreiben lernen: Eine Einführung. Darmstadt: WBG.

Grieshammer, E., Liebetanz, F., Peters, N., \& Zegenhagen, J. (2016). Zukunftsmodell Schreibberatung: Eine Anleitung zur Begleitung von Schreibenden im Studium (3., korrigierte Aufl.). Baltmannsweiler: Schneider Verlag Hohengehren.

Günther, K. (2020). Selbstcoaching in der Wissenschaft: Wie das Schreiben gelingt. Opladen: Barbara Budrich.

Hayes, J. R. (2014). Kognition und Affekt beim Schreiben: Ein neues Konzept. In S. Dreyfürst \& N. Sennewald (Hrsg.), Schreiben: Grundlagentexte zur Theorie, Didaktik und Beratung (S. 87-94). Opladen: Barbara Budrich.

Kaiser, A. (2015). Reiseführer für die Unikarriere: Zwischen Schlangengrube und Wissenschaftsoase. Opladen: Barbara Budrich.

Keding, G., \& Scharlau, I. (2016). Drei Einflüsse auf Schreibberatung und ein integriertes Modell der Selbststeuerung für Schreibberaterinnen und Schreibberater. In S. Ballweg (Hrsg.), Schreibberatung und Schreibförderung: Impulse aus Theorie und Empirie und Praxis (S. 115-134). Frankfurt/M.: Peter Lang.

Kellogg, R. T. (2014). Schreibkompetenzen schulen. Eine Perspektive der kognitiven Entwicklungspsychologie. In S. Dreyfürst \& N. Sennewald (Hrsg.), Schreiben: Grundlagentexte zur Theorie, Didaktik und Beratung (S. 127-151). Opladen: Barbara Budrich.

Klemm, M. (2004). Schreibberatung und Schreibtraining. In K. Knapp, Antos, M. G: Becker-Mrotzek, A. Deppermann, S. Göpferich, J. Grabowski, M. Klemm \& C. Villiger (Hrsg.), Angewandte Linguistik: Ein Lehrbuch (S. 120-142). Tübingen: Francke.

König, E., \& Volmer, G. (2019). Handbuch Systemisches Coaching: Für Coaches und Führungskräfte, Berater und Trainer (3. Aufl.). Weinheim: Beltz.

Kruse, O. (2007). Keine Angst vor dem leeren Blatt: Ohne Schreibblockaden durchs Studium (12. Aufl.). Frankfurt/M.: Campus.

Kruse, O., \& Chitez, M. (2014). Schreibkompetenz im Studium: Komponenten, Modelle und Assessment. In S. Dreyfürst \& N. Sennewald (Hrsg.), Schreiben: Grundlagentexte zur Theorie, Didaktik und Beratung (S. 107-126). Opladen: Barbara Budrich.

Kruse, O., \& Ruhmann, G. (2006). Prozessorientierte Schreibdidaktik: Eine Einführung. In O. Kruse, K. Berger \& M. Ulmi (Hrsg.), Prozessorientierte Schreibdidaktik: Schreibtraining für Schule, Studium und Beruf (S. 13-35). Bern: Haupt.

Kuhn, C., Kühl, F., Kimmerle, L. L., Hertweck, L., Drumm, S., Hampe, M., \& Schabel, S. (2016). Wie können Studierende an die universitäre Textproduktion im Bachelorstudium herangeführt werden? Konzeption und Hintergrund zweier Lehrveranstaltungen an der Technischen Universität Darmstadt. In M. Lichtlein, V. Fritsch, R. Graßmann, K. Hiltmann \& M. Kraft (Hrsg.), Interdisziplinäre Konzepte: Akademisches Schreiben in den Natur- und Ingenieurwissenschaften (S. 54-71). Coburg: Aumann.

Kuntschner, E. (2020). Schreibcoaching und Schreibberatung - der Versuch einer Abgrenzung. Coaching | Theorie \& Praxis. https:// doi.org/10.1365/s40896-020-00049-7.

Lange, U., \& Wiethoff, M. (2014). Systemische Schreibberatung. In S. Dreyfürst \& N. Sennewald (Hrsg.), Schreiben: Grundlagentexte zur Theorie, Didaktik und Beratung (S. 283-301). Opladen: Barbara Budrich.

Nußbeck, S. (2010). Einführung in die Beratungspsychologie: Mit 94 Übungsfragen und 3 Tabellen (2. Aufl.). München: Reinhardt.

Par, A., Starke-Meyerring, D., \& McAlpine, L. (2009). The dissertation as multi-genre: many readers, many readings: many readers, many readings. In C. Bazerman, A. Bonini \& D. Figueiredo (Hrsg.), Genre in a changing world (S. 183-197). Fort Collings, West Lafayette: Parlor Press, The WAC Clearinghouse. https://doi.org/ 10.37514/PER-B.2009.2324.2.09.

Rabe, F. (2016). Sprachliche und fachliche Anforderungen an Wissenschaftler aus verschiedenen Disziplinen. In A. Hirsch-Weber \& S. Scherer (Hrsg.), Wissenschaftliches Schreiben in Natur- und Technikwissenschaften: Neue Herausforderungen der Schreibforschung (S. 55-83). Wiesbaden: Springer Spektrum. 
Reimers, U. (2020). The "unwritten law" of language choice in STEM doctoral dissertations: a case study in the German-speaking context. JoSch - Journal der Schreibberatung, 1(9), 10-21.

Rogers, C.R. (1972). Die nicht-direktive Beratung: Counseling and psychotherapy. München: Kindler.

Schreyögg, A. (2010). Ist Coaching reine Prozessberatung oder sind hier auch andere Beratungsmodelle relevant? Organisationsberatung, Supervision, Coaching, 17(2), 119-132. https://doi.org/10. 1007/s11613-010-0185-x.

Schreyögg, A. (2012). Coaching: Eine Einführung für Praxis und Ausbildung (7. Aufl.). Frankfurt/M.: Campus.
Webers, T. (2020). Systemisches Coaching. Berlin, Heidelberg: Springer. https://doi.org/10.1007/978-3-662-61336-8.

Wetschanow, K., \& Fleischhacker, M. (2020). Coaching mit Schreiben oder Schreiben mit Coaching? - Zum Start des Themenschwerpunktes „Interventionen im Schreibcoaching“. Coaching I Theorie \& Praxis, 6(1), 155-161. https://doi.org/10.1365/s40896-02000042-0.

Publisher's Note Springer Nature remains neutral with regard to jurisdictional claims in published maps and institutional affiliations. 